\title{
WEIL-PETERSSON AND LITTLE TEICHMÜLLER SPACES ON THE REAL LINE
}

\author{
Yuliang Shen, Shuan Tang* and Li Wu \\ Soochow University, Department of Mathematics \\ Suzhou 215006, P. R. China; ylshen@suda.edu.cn \\ Guizhou Normal University, School of Mathematics Sciences \\ Guiyang 550001, P. R. China; tsa@gznu.edu.cn \\ Soochow University, Department of Mathematics \\ Suzhou 215006, P. R. China; wuli187@126.com
}

\begin{abstract}
We will prove that an increasing homeomorphism $h$ in the Weil-Petersson class on the real line must be locally absolutely continuous such that $\log h^{\prime}$ belongs to the Sobolev class $H^{\frac{1}{2}}$. We will also deal with the the pre-logarithmic derivative models of the little and Weil-Petersson Teichmüller spaces in the half plane case.
\end{abstract}

\section{Introduction}

This is a continuous work of our previous papers [Sh] and [ST] (see also [HWS]), where we were mainly concerned with the Weil-Petersson geometry theory of the universal Teichmüller space. We first fix some basic notations. Let $\mathbf{U}=\{z=$ $x+i y: y>0\}$ and $\mathbf{U}^{*}=\{z=x+i y: y<0\}$ denote the upper and lower half plane in the complex plane $\mathbf{C}$, respectively. $\mathbf{R}=\partial \mathbf{U}=\partial \mathbf{U}^{*}$ is the real line, and $\hat{\mathbf{R}}=\mathbf{R} \cup\{\infty\}$ is the extended real line in the Riemann sphere $\hat{\mathbf{C}}=\mathbf{C} \cup\{\infty\}$. Let $\Delta=\{z:|z|<1\}$ denote the unit disk. $\Delta^{*}=\hat{\mathbf{C}}-\bar{\Delta}$ is the exterior of $\Delta$, and $S^{1}=\partial \Delta=\partial \Delta^{*}$ is the unit circle. $\mathbf{D}$ will always denote the unit disk $\Delta$ or the upper half plane $\mathbf{U}$ so that $\mathbf{S}=\partial \mathbf{D}$ is the unit circle $S^{1}$ or the real line $\mathbf{R}$. Similarly, $\mathbf{D}^{*}$ will always denote the exterior $\Delta^{*}$ of the unit disk or the lower half plane $\mathbf{U}^{*}$.

A sense-preserving homeomorphism of $\mathbf{S}$ onto itself is said to belong to the WeilPetersson class, which is denoted by $\mathrm{WP}(\mathbf{S})$, if it has a quasiconformal extension to D whose Beltrami coefficient is square integrable in the Poincaré metric. In [Sh], we gave the following intrinsic characterization of a quasisymmetric homeomorphism in the Weil-Petersson class $\mathrm{WP}\left(S^{1}\right)$ without using quasiconformal extensions, which solves a problem proposed by Takhtajan-Teo in 2006 (see page 68 in [TT] and also [Fi], [GR]). Recall that, for a function $f$ defined on $\mathbf{S}, f^{\prime}$ denotes the derivative of $f$, namely, for $z \in \mathbf{S}$,

$$
f^{\prime}(z) \doteq \lim _{\mathbf{S} \ni \zeta \rightarrow z} \frac{f(\zeta)-f(z)}{\zeta-z}
$$

provided the limit exists, while $f^{\prime}(z) \doteq 0$ otherwise.

https://doi.org/10.5186/aasfm.2018.4358

2010 Mathematics Subject Classification: Primary 30C62, 30F60, 32G15.

Key words: Universal Teichmüller space, little Teichmüller space, Weil-Petersson Teichmüller space, Weil-Petersson class, quasiconformal mapping, Sobolev class.

${ }^{*}$ The first author.

Research supported by the National Natural Science Foundation of China (Grant Nos. 11601100, 11631010). 
Theorem 1.1. [Sh] A sense-preserving homeomorphism $g$ on the unit circle $S^{1}$ belongs to the Weil-Petersson class $\mathrm{WP}\left(S^{1}\right)$ if and only if $g$ is absolutely continuous (with respect to the arc-length measure) such that $\log g^{\prime}$ belongs to the Sobolev class $H^{\frac{1}{2}}\left(S^{1}\right)$.

Recall that the Sobolev class $H^{\frac{1}{2}}(\mathbf{S})$ on $\mathbf{S}$ is the collection of all locally integrable functions $u$ with

$$
\|u\|_{H^{\frac{1}{2}}}^{2} \doteq \frac{1}{4 \pi^{2}} \int_{\mathbf{S}} \int_{\mathbf{S}} \frac{|u(\zeta)-u(\eta)|^{2}}{|\zeta-\eta|^{2}}|d \zeta \| d \eta|<+\infty .
$$

Since the logarithmic derivative is not invariant under a Möbius transformation, we can not deduce a similar result on the real line from Theorem 1.1 directly. Anyway, by means of a construction due to Semmes (see [Se1-2]), we did prove the following result in our joint work [ST].

Theorem 1.2. [ST] Let $h$ be an increasing homeomorphism from the real line $\mathbf{R}$ onto itself. If $h$ is locally absolutely continuous with $\log h^{\prime} \in H^{\frac{1}{2}}(\mathbf{R})$, then $h \in$ $\mathrm{WP}(\mathbf{R})$.

In this note, we will show that the converse to Theorem 1.2 is also true, namely, we have

Theorem 1.3. Let $h$ be an increasing homeomorphism from the real line $\mathbf{R}$ onto itself such that $h \in \mathrm{WP}(\mathbf{R})$. Then $h$ is locally absolutely continuous with $\log h^{\prime} \in H^{\frac{1}{2}}(\mathbf{R})$.

To prove Theorem 1.3, we will deal with the pre-logarithmic derivative models of the little and Weil-Petersson Teichmüller spaces in the half plane case. It should be pointed out that, in the unit disk case, the pre-logarithmic derivative models of the little and Weil-Petersson Teichmüller spaces were much investigated in recent years (see Propositions 3.1 and 4.1 below).

\section{Preliminaries}

In this section, we give some basic definitions and results on the universal Teichmüller space and its two subspaces, the little Teichmüller space and the WeilPetersson Teichmüller space. In particular, we will recall the Schwarzian derivative models of these Teichmüller spaces.

We begin with the standard theory of the universal Teichmüller space (see [Ah], [GL], [Le] and [Na] for more details). Let $M\left(\mathbf{D}^{*}\right)$ denote the open unit ball of the Banach space $L^{\infty}\left(\mathbf{D}^{*}\right)$ of essentially bounded measurable functions on $\mathbf{D}^{*}$. For $\mu \in M\left(\mathbf{D}^{*}\right)$, let $f_{\mu}$ be the quasiconformal mapping on the extended plane $\hat{\mathbf{C}}$ with complex dilatation equal to $\mu$ in $\mathbf{D}^{*}$, conformal in $\mathbf{D}$, normalized by $f_{\mu}(0)=0$, $f_{\mu}(1)=1$ and $f_{\mu}(\infty)=\infty$. We say two elements $\mu$ and $\nu$ in $M\left(\mathbf{D}^{*}\right)$ are equivalent, denoted by $\mu \sim \nu$, if $\left.f_{\mu}\right|_{\mathbf{D}}=\left.f_{\nu}\right|_{\mathbf{D}}$. Then $T=M\left(\mathbf{D}^{*}\right) / \sim$ is the Bers model of the universal Teichmüller space. We let $\Phi$ denote the natural projection from $M\left(\mathbf{D}^{*}\right)$ onto $T$ so that $\Phi(\mu)$ is the equivalence class $[\mu]$. [0] is called the base point of $T$.

Let $\Omega$ be an arbitrary simply connected domain in the extended complex plane $\hat{\mathbf{C}}$ which is conformally equivalent to the upper half plane. Recall that the hyperbolic metric $\lambda_{\Omega}$ (with curvature constantly equal to -4 ) in $\Omega$ can be defined by

$$
\lambda_{\Omega}(f(z))\left|f^{\prime}(z)\right|=\frac{1}{2 y}, \quad z=x+i y \in \mathbf{U}
$$


where $f: \mathbf{U} \rightarrow \Omega$ is any conformal mapping. Let $B(\Omega)$ denote the Banach space of functions $\phi$ holomorphic in $\Omega$ with norm

$$
\|\phi\|_{B(\Omega)} \doteq \sup _{z \in \Omega}|\phi(z)| \lambda_{\Omega}^{-2}(z)
$$

and $B_{0}(\Omega)$ the closed subspace of $B(\Omega)$ which consists of those functions $\phi$ such that

$$
\inf \left\{\sup _{z \in \Omega \backslash K}|\phi(z)| \lambda_{\Omega}^{-2}(z): K \subset \Omega \text { compact }\right\}=0 .
$$

We also denote by $B^{*}(\Omega)$ the Banach space of functions $\phi$ holomorphic in $\Omega$ with finite norm

$$
\|\phi\|_{B^{*}(\Omega)} \doteq\left(\frac{1}{\pi} \iint_{\Omega}|\phi(z)|^{2} \lambda_{\Omega}^{-2} d x d y\right)^{\frac{1}{2}} .
$$

Then, $B^{*}(\Omega) \subset B_{0}(\Omega)$, and the inclusion map is continuous (see [Zh]). It is easy to see that a conformal mapping $g: \Omega_{1} \rightarrow \Omega_{2}$ induces a map $g^{*}: \phi \mapsto(\phi \circ g)\left(g^{\prime}\right)^{2}$, which are isometric isomorphisms from $B\left(\Omega_{2}\right)$ onto $B\left(\Omega_{1}\right)$, from $B_{0}\left(\Omega_{2}\right)$ onto $B_{0}\left(\Omega_{1}\right)$, and from $B^{*}\left(\Omega_{2}\right)$ onto $B^{*}\left(\Omega_{1}\right)$.

It is known that the universal Teichmüller space $T$ is an infinite dimensional complex Banach manifold. To make this precise, we consider the map $S: M\left(\mathbf{D}^{*}\right) \rightarrow$ $B(\mathbf{D})$ which sends $\mu$ to the Schwarzian derivative of $\left.f_{\mu}\right|_{\mathbf{D}}$. Recall that for any locally univalent function $f$, its Schwarzian derivative $S_{f}$ is defined by

$$
S_{f} \doteq N_{f}^{\prime}-\frac{1}{2} N_{f}^{2}, \quad N_{f} \doteq\left(\log f^{\prime}\right)^{\prime} .
$$

$S$ is a holomorphic split submersion onto its image, which descends down to a map $\beta: T \rightarrow B(\mathbf{D})$ known as the Bers embedding. Via the Bers embedding, $T$ carries a natural complex Banach manifold structure so that $\Phi$ is a holomorphic split submersion.

Let $L_{0}\left(\mathbf{D}^{*}\right)$ be the closed subspace of $L^{\infty}\left(\mathbf{D}^{*}\right)$ which consists of those functions $\mu$ such that

$$
\inf \left\{\left\|\left.\mu\right|_{\mathbf{D}^{*} \backslash K}\right\|_{\infty}: K \subset \mathbf{D}^{*} \text { compact }\right\}=0 .
$$

Set $M_{0}\left(\mathbf{D}^{*}\right)=M\left(\mathbf{D}^{*}\right) \cap L_{0}\left(\mathbf{D}^{*}\right)$. Then $T_{0}=M_{0}\left(\mathbf{D}^{*}\right) / \sim$ is called the little Teichmüller space. Under the Bers embedding $S: M\left(\mathbf{D}^{*}\right) \rightarrow B(\mathbf{D}), S\left(M_{0}\left(\mathbf{D}^{*}\right)=S\left(M\left(\mathbf{D}^{*}\right)\right) \cap\right.$ $B_{0}(\mathbf{D})$ (see [GL], [GS], [Po]).

We proceed to define the Weil-Petersson Teichmüller space. We denote by $L^{*}\left(\mathbf{D}^{*}\right)$ the Banach space of all essentially bounded measurable functions $\mu$ on $\mathbf{D}^{*}$ with norm

$$
\|\mu\|_{\mathrm{WP}} \doteq\|\mu\|_{\infty}+\left(\frac{1}{\pi} \iint_{\mathbf{D}^{*}}|\mu(z)|^{2} \lambda_{\mathbf{D}^{*}}^{2}(z) d x d y\right)^{\frac{1}{2}} .
$$

Set $M^{*}\left(\mathbf{D}^{*}\right)=M\left(\mathbf{D}^{*}\right) \cap L^{*}\left(\mathbf{D}^{*}\right)$. Then $T^{*}=M^{*}\left(\mathbf{D}^{*}\right) / \sim$ is known as the WeilPetersson Teichmüller space. Actually, $T^{*}$ is the base point component of the universal Teichmüller space under the complex Hilbert manifold structure introduced by Takhtajan-Teo [TT]. Under the Bers projection $S: M\left(\mathbf{D}^{*}\right) \rightarrow B(\mathbf{D}), S\left(M^{*}\left(\mathbf{D}^{*}\right)\right)=$ $S\left(M\left(\mathbf{D}^{*}\right)\right) \cap B^{*}(\mathbf{D})($ see $[\mathrm{Cu}],[\mathrm{TT}])$. 


\section{Pre-logarithmic derivative model of the little Teichmüller space}

The universal Teichmüller space has another important model, the pre-logarithmic derivative model (see [AG], [Zhu]). In the unit disk case, the pre-logarithmic derivative models of the little and Weil-Petersson Teichmüller spaces were much investigated in recent years (see Propositions 3.1 and 4.1 below, and see [Cu], [GL], [GS], [Po], [Sh], [TT] for more details). Contrary to the Schwarzian derivative model, the logarithmic derivative is not invariant under a Möbius transformation. Therefore, we can not transfer Propositions 3.1 and 4.1 directly to the half plane case. In this and next sections, we will deal with the pre-logarithmic derivative models of the little and Weil-Petersson Teichmüller spaces in the half plane case. The results will be used to prove Theorem 1.3 and have independent interests of their own.

Let $\mathcal{B}(\mathbf{D})$ denote the Bloch space of functions $\phi$ holomorphic in $\mathbf{D}$ with semi-norm

$$
\|\phi\|_{\mathcal{B}(\mathbf{D})} \doteq \sup _{z \in \mathbf{D}}\left|\phi^{\prime}(z)\right| \lambda_{\mathbf{D}}^{-1}(z),
$$

and $\mathcal{B}_{0}(\mathbf{D})$ the subspace of $\mathcal{B}(\mathbf{D})$ which consists of those functions $\phi$ such that

$$
\inf \left\{\sup _{z \in \mathbf{D} \backslash K}\left|\phi^{\prime}(z)\right| \lambda_{\mathbf{D}}^{-1}(z): K \subset \mathbf{D} \text { compact }\right\}=0 .
$$

It is known that, for each holomorphic function $\phi$ on $\mathbf{D}, \phi \in \mathcal{B}(\mathbf{D})$ if and only if $\phi^{\prime \prime} \in B(\mathbf{D})($ see $[\mathrm{Zh}])$.

Koebe distortion theorem implies that $\left.\log f_{\mu}^{\prime}\right|_{\mathbf{D}} \in \mathcal{B}(\mathbf{D})$ for $\mu \in M\left(\mathbf{D}^{*}\right)$. Furthermore, the map $L$ induced by the correspondence $\left.\mu \mapsto \log f_{\mu}^{\prime}\right|_{\mathbf{D}}$ is a continuous map from $M\left(\mathbf{D}^{*}\right)$ into $\mathcal{B}(\mathbf{D})$ (see [Le]). Actually, $L: M\left(\mathbf{D}^{*}\right) \rightarrow \mathcal{B}(\mathbf{D})$ is even holomorphic (see $[\mathrm{Ha}])$.

We recall the following known result (see [GL], [GS], [Po]).

Proposition 3.1. Given $\mu \in M\left(\Delta^{*}\right)$, the following statements are all equivalent:

(1) There exists some $\nu \in M_{0}\left(\Delta^{*}\right)$ such that $[\nu]=[\mu]$;

(2) $L(\mu) \in \mathcal{B}_{0}(\Delta)$

(3) $S(\mu) \in B_{0}(\Delta)$.

Now we show a similar result in the half plane case.

Theorem 3.2. Given $\mu \in M\left(\mathbf{U}^{*}\right)$, the following statements are all equivalent:

(1) There exists some $\nu \in M_{0}\left(\mathbf{U}^{*}\right)$ such that $[\nu]=[\mu]$;

(2) $L(\mu) \in \mathcal{B}_{0}(\mathbf{U})$

(3) $S(\mu) \in B_{0}(\mathbf{U})$.

Proof. As stated in section 2, (1) $\Leftrightarrow(3)$ is known (see [GL], [GS], [Po]). We need to show that $(1) \Rightarrow(2) \Rightarrow(3)$.

To show $(1) \Rightarrow(2)$, we use the continuity of the map $L: M\left(\mathbf{D}^{*}\right) \rightarrow \mathcal{B}(\mathbf{D})$. Let $\mu \in M_{0}\left(\mathbf{U}^{*}\right)$ be given. Set $\mathbf{U}_{n}^{*}=\{x+i y:-n<x<n,-n<y<-1 / n\}$ for each positive integer $n$, and consider

$$
\mu_{n}(z)= \begin{cases}\mu(z), & z \in \mathbf{U}_{n}^{*}, \\ 0, & z \in \mathbf{U}^{*} \backslash \mathbf{U}_{n}^{*} .\end{cases}
$$

Then $\left\|\mu_{n}-\mu\right\|_{\infty} \rightarrow 0$ as $n \rightarrow \infty$. By the continuity of $L: M\left(\mathbf{U}^{*}\right) \rightarrow \mathcal{B}(\mathbf{U})$ we obtain $\left\|L\left(\mu_{n}\right)-L(\mu)\right\|_{\mathcal{B}(\mathbf{U})} \rightarrow 0$ as $n \rightarrow \infty$. On the other hand, since $f_{\mu_{n}}$ is conformal outside $\mathbf{U}_{n}^{*}$, it is obvious that $L\left(\mu_{n}\right) \in \mathcal{B}_{0}(\mathbf{U})$. Since $\mathcal{B}_{0}(\mathbf{U})$ is closed in $\mathcal{B}(\mathbf{U})$, we conclude that $L(\mu) \in \mathcal{B}_{0}(\mathbf{U})$ as desired. 
Noting that

$$
S(\mu)=L^{\prime \prime}(\mu)-\frac{1}{2}\left(L^{\prime}(\mu)\right)^{2},
$$

we conclude that $(2) \Rightarrow(3)$ follows immediately from the following lemma. Here and in what follows, we use $L^{\prime}(\mu)$ and $L^{\prime \prime}(\mu)$ respectively to denote the first and second order derivatives of $L(\mu)$.

Lemma 3.3. Let $\phi$ be a holomorphic function on the upper half plane $\mathbf{U}$ such that $\phi \in \mathcal{B}_{0}(\mathbf{U})$. Then $\phi^{\prime \prime} \in B_{0}(\mathbf{U})$.

Proof. For $z=x+i y \in \mathbf{U}$, it follows from the Cauchy integral formula that

$$
\left|\phi^{\prime \prime}(z)\right|=\left|\frac{1}{2 \pi i} \int_{|\zeta-z|=y / 4} \frac{\phi^{\prime}(\zeta)}{(\zeta-z)^{2}} d \zeta\right| \leq 4 / y \sup _{|\zeta-z|<y / 4}\left|\phi^{\prime}(\zeta)\right| .
$$

Noting that the function $\left|\phi^{\prime}\right|^{2}$ is subharmonic in $\mathbf{U}$, we have

$$
\left|\phi^{\prime}(\zeta)\right|^{2} \leq \frac{16}{\pi y^{2}} \iint_{|w-\zeta|<y / 4}\left|\phi^{\prime}(w)\right|^{2} d u d v, \quad|\zeta-z|<y / 4 .
$$

Combing (3.3) with (3.4) we obtain

$$
y^{4}\left|\phi^{\prime \prime}(z)\right|^{2} \leq \frac{256}{\pi} \int_{y / 2<v<2 y} \int_{|u-x|<y}\left|\phi^{\prime}(w)\right|^{2} d u d v .
$$

Here and in what follows, we will always denote

$$
\mathbf{U}(t)=\{x+i y:-1 / t<x<1 / t, t<y<1 / t\}, \quad 0<t<1 .
$$

Since $\phi \in \mathcal{B}_{0}(\mathbf{U})$, we conclude that for every $\epsilon>0$, there is some $t_{0}>0$ such that

$$
v\left|\phi^{\prime}(w)\right|<\frac{\sqrt{\pi \epsilon}}{16 \sqrt{3}}, \quad w=u+i v \in \mathbf{U} \backslash \mathbf{U}\left(t_{0}\right) .
$$

Now we let $z=x+i y \in \mathbf{U} \backslash \mathbf{U}\left(t_{0} / 5\right)$. It is easy to see that $w=u+i v \in \mathbf{U} \backslash \mathbf{U}\left(t_{0}\right)$ whenever $y / 2<v<2 y$ and $|u-x|<y$. Thus it follows from (3.5) and (3.7) that

$$
y^{4}\left|\phi^{\prime \prime}(z)\right|^{2} \leq \frac{\epsilon}{3} \int_{y / 2<v<2 y} \int_{|u-x|<y} v^{-2} d u d v=\epsilon,
$$

which implies that $\phi^{\prime \prime} \in B_{0}(\mathbf{U})$.

\section{Pre-logarithmic derivative model of the Weil-Petersson Teichmüller space}

We now consider the pre-logarithmic derivative model of the Weil-Petersson Teichmüller space. Let $\mathcal{D}(\mathbf{D})$ denote the Dirichlet space of functions $\phi$ holomorphic in $\mathrm{D}$ with semi-norm

$$
\|\phi\|_{\mathcal{D}(\mathbf{D})} \doteq\left(\frac{1}{\pi} \iint_{\mathbf{D}}\left|\phi^{\prime}(z)\right|^{2} d x d y\right)^{\frac{1}{2}} .
$$

It is known that, for each holomorphic function $\phi$ on $\mathbf{D}, \phi \in \mathcal{D}(\mathbf{D})$ if and only if $\phi^{\prime \prime} \in B^{*}(\mathbf{D})$. It is also known that $\mathcal{D}(\mathbf{D}) \subset \mathcal{B}_{0}(\mathbf{D})$, and the inclusion map is continuous (see $[\mathrm{Zh}]$ ).

We have the following known result (see [Cu], [Sh], [TT]).

Proposition 4.1. Given $\mu \in M\left(\Delta^{*}\right)$, the following statements are all equivalent:

(1) There exists some $\nu \in M^{*}\left(\Delta^{*}\right)$ such that $[\nu]=[\mu]$; 
(2) $L(\mu) \in \mathcal{D}(\Delta)$;

(3) $S(\mu) \in B^{*}(\Delta)$.

To obtain a similar result in the half plane case, we need two lemmas. The first is a Hardy-type inequality (see $[\mathrm{KP}]$ ).

Lemma 4.2. Let $1<p \leq q<\infty$, and $u(s), v(s)$ be two positive measurable functions in the interval $(a, b)$. If

$$
A \doteq \sup _{a<x<b}\left(\int_{a}^{x} u(s) d s\right)^{1 / q}\left(\int_{x}^{b} v(s)^{1-p^{\prime}} d s\right)^{1 / p^{\prime}}<\infty,
$$

where $p^{\prime}=p /(p-1)$. Then there is constant $C(p, q)>0$ such that for all positive measurable functions $f$ in the interval $(a, b)$, the following inequality holds

$$
\left(\int_{a}^{b}\left(\int_{x}^{b} f(s) d s\right)^{q} u(x) d x\right)^{1 / q} \leq C(p, q) A\left(\int_{a}^{b} f(x)^{p} v(x) d x\right)^{1 / p} .
$$

Lemma 4.3. Let $\phi$ be a holomorphic function on the upper half plane $\mathbf{U}$ such that $\phi^{\prime} \in B(\mathbf{U})$ and $\lim _{y \rightarrow \infty} \phi(x+i y)=0$ uniformly for $x \in \mathbf{R}$. Then there exists some universal constant $C>0$ such that

$$
\iint_{\mathbf{U}_{t}}|\phi(x+i y)|^{2} d x d y \leq C\left(\iint_{\mathbf{U}_{t}}\left|\phi^{\prime}(x+i y)\right|^{2} y^{2} d x d y+\left\|\phi^{\prime}\right\|_{B(\mathbf{U})}^{2}\right) .
$$

Proof. By assumption we have

$$
\phi(x+i y)=-i \int_{y}^{\infty} \phi^{\prime}(x+i v) d v
$$

which implies that

$$
|\phi(x+i y)| \leq \int_{y}^{\infty}\left|\phi^{\prime}(x+i v)\right| d v \leq \int_{y}^{1 / t}\left|\phi^{\prime}(x+i v)\right| d v+\frac{\left\|\phi^{\prime}\right\|_{B(\mathbf{U})}}{4} t .
$$

Then

$$
\int_{t}^{1 / t}|\phi(x+i y)|^{2} d y \leq 2 \int_{t}^{1 / t}\left(\int_{y}^{1 / t}\left|\phi^{\prime}(x+i v)\right| d v\right)^{2} d y+\frac{\left\|\phi^{\prime}\right\|_{B(\mathbf{U})}^{2}}{8} t .
$$

By Lemma 4.2 with $u(s)=1, v(s)=s^{2}$, and $p=q=2$, we conclude that there is a universal constant $C>0$ such that

$$
\int_{t}^{1 / t}\left(\int_{y}^{1 / t}\left|\phi^{\prime}(x+i v)\right| d v\right)^{2} d y \leq C A(t) \int_{t}^{1 / t}\left|\phi^{\prime}(x+i y)\right|^{2} y^{2} d y
$$

where

$$
A(t)=\sup _{t<x<1 / t}|x-t|^{1 / 2}|1 / x-t|^{1 / 2}=1-t \leq 1
$$

So we have

$$
\int_{t}^{1 / t}|\phi(x+i y)|^{2} d y \leq 2 C \int_{t}^{1 / t}\left|\phi^{\prime}(x+i y)\right|^{2} y^{2} d y+\frac{\left\|\phi^{\prime}\right\|_{B(\mathbf{U})}^{2}}{8} t .
$$

Integrating both sides of the above inequality with respect to $x$ from $-1 / t$ to $1 / t$, we get

$$
\iint_{\mathbf{U}_{t}}|\phi(x+i y)|^{2} d x d y \leq 2 C \iint_{\mathbf{U}_{t}}\left|\phi^{\prime}(x+i y)\right|^{2} y^{2} d x d y+\frac{\left\|\phi^{\prime}\right\|_{B(\mathbf{U})}^{2}}{4} .
$$


This completes the proof of the lemma.

Now we can prove the main result of this section.

Theorem 4.4. Given $\mu \in M\left(\mathbf{U}^{*}\right)$, the following statements are all equivalent:

(1) There exists some $\nu \in M^{*}\left(\mathbf{U}^{*}\right)$ such that $[\nu]=[\mu]$;

(2) $L(\mu) \in \mathcal{D}(\mathbf{U})$;

(3) $S(\mu) \in B^{*}(\mathbf{U})$.

Proof. As stated in Section 2, (1) $\Leftrightarrow(3)$ is known (see [Cu], [TT]). Recall that, for each holomorphic function $\phi$ on $\mathbf{U}, \phi \in \mathcal{D}(\mathbf{U})$ if and only if $\phi^{\prime \prime} \in B^{*}(\mathbf{U})$, and $\mathcal{D}(\mathbf{U}) \subset \mathcal{B}_{0}(\mathbf{U})$, we find out that (2) $\Rightarrow(3)$ follows immediately from (3.2). Precisely,

$$
\begin{aligned}
\iint_{\mathbf{U}}|S(\mu)(x+i y)|^{2} y^{2} d x d y \leq & 2 \iint_{\mathbf{U}}\left|L^{\prime \prime}(\mu)(x+i y)\right|^{2} y^{2} d x d y \\
& +\iint_{\mathbf{U}}\left|L^{\prime}(\mu)(x+i y)\right|^{4} y^{2} d x d y \\
\leq & \pi\left\|L^{\prime \prime}(\mu)\right\|_{B^{*}(\mathbf{U})}^{2}+\pi\|L(\mu)\|_{\mathcal{B}(\mathbf{U})}^{2}\|L(\mu)\|_{\mathcal{D}(\mathbf{U})}^{2}<\infty .
\end{aligned}
$$

It remains to show that $(3) \Rightarrow(2)$. We assume that $S(\mu) \in B^{*}(\mathbf{U})$ so that $S(\mu) \in B_{0}(\mathbf{U})$, which implies by Theorem 3.2 that $L(\mu) \in \mathcal{B}_{0}(\mathbf{U})$. Fix some $\epsilon>0$ so small such that $\epsilon<1 / C$, where $C>0$ is the constant in Lemma 4.3. Then there is a positive constant $t_{0}<1$ such that for all $z=x+i y \in \mathbf{U} \backslash \mathbf{U}\left(t_{0}\right)$,

$$
y^{2}\left|L^{\prime}(\mu)(x+i y)\right|^{2}<\epsilon .
$$

By Lemma 4.3 we have for $0<t<t_{0}$ that

$$
\begin{aligned}
& 1 / C \iint_{\mathbf{U}(t)}\left|L^{\prime}(\mu)(x+i y)\right|^{2} d x d y \\
& \leq \iint_{\mathbf{U}(t)}\left|L^{\prime \prime}(\mu)(x+i y)\right|^{2} y^{2} d x d y+\left\|L^{\prime \prime}(\mu)\right\|_{B(\mathbf{U})}^{2} \\
& \leq 2 \iint_{\mathbf{U}(t)}|S(\mu)(x+i y)|^{2} y^{2} d x d y+1 / 2 \iint_{\mathbf{U}(t)}\left|L^{\prime}(\mu)(x+i y)\right|^{4} y^{2} d x d y+\left\|L^{\prime \prime}(\mu)\right\|_{B(\mathbf{U})}^{2} \\
& \leq 2 \pi\|S(\mu)\|_{B^{*}(\mathbf{U})}^{2}+1 / 2 \iint_{\mathbf{U}\left(t_{0}\right)}\left|L^{\prime}(\mu)(x+i y)\right|^{4} y^{2} d x d y \\
& \quad+1 / 2 \iint_{\mathbf{U}(t) \backslash \mathbf{U}\left(t_{0}\right)}\left|L^{\prime}(\mu)(x+i y)\right|^{4} y^{2} d x d y+\left\|L^{\prime \prime}(\mu)\right\|_{B(\mathbf{U})}^{2} \\
& \leq 1 / 2 \iint_{\mathbf{U}\left(t_{0}\right)}\left|L^{\prime}(\mu)(x+i y)\right|^{4} y^{2} d x d y+\epsilon / 2 \iint_{\mathbf{U}(t)}\left|L^{\prime}(\mu)(x+i y)\right|^{2} d x d y \\
& \quad+2 \pi\|S(\mu)\|_{B^{*}(\mathbf{U})}^{2}+\left\|L^{\prime \prime}(\mu)\right\|_{B(\mathbf{U})}^{2},
\end{aligned}
$$

which implies that

$$
\begin{aligned}
& (1 / C-\epsilon / 2) \iint_{\mathbf{U}(t)}\left|L^{\prime}(\mu)(x+i y)\right|^{2} d x d y \\
& \leq 1 / 2 \iint_{\mathbf{U}\left(t_{0}\right)}\left|L^{\prime}(\mu)(x+i y)\right|^{4} y^{2} d x d y+2 \pi\|S(\mu)\|_{B^{*}(\mathbf{U})}^{2}+\left\|L^{\prime \prime}(\mu)\right\|_{B(\mathbf{U})}^{2}<\infty .
\end{aligned}
$$

Letting $t \rightarrow 0$ we obtain $L(\mu) \in \mathcal{D}(\mathbf{U})$ as desired. 


\section{Proof of Theorem 1.3}

To prove Theorem 1.3, we need a basic result concerning the pull-back operator on the Sobolev space $H^{\frac{1}{2}}(\mathbf{S})$ by a quasisymmetric homeomorphism. Recall that a sense-preserving homeomorphism $h$ from $\mathbf{S}$ onto itself is said to be quasisymmetric if there exists a positive constant $C$ such that $\left|h\left(I_{1}\right)\right| \leq C\left|h\left(I_{2}\right)\right|$ for all pairs of adjacent intervals $I_{1}$ and $I_{2}$ on $\mathbf{S}$ with the same length $\left|I_{1}\right|=\left|I_{2}\right|$. Beurling-Ahlfors [BA] proved that a sense-preserving homeomorphism $h$ from $\mathbf{S}$ onto itself is quasisymmetric if and only if there exists some quasiconformal homeomorphism of $\mathbf{D}$ onto itself which has boundary values $h$. We have the following well-known result (see [BA], [NS]).

Proposition 5.1. Let $h$ be a sense-preserving homeomorphism from $\mathbf{S}$ onto itself. Then the pull-back operator $P_{h}$ defined by $P_{h} u=u \circ h$ is a bounded operator from $H^{\frac{1}{2}}(\mathbf{S})$ into itself if and only if $h$ is quasisymmetric.

Proof of Theorem 1.3. Let $h$ be an increasing homeomorphism from the real line $\mathbf{R}$ onto itself such that $h \in \mathrm{WP}(\mathbf{R})$. Then $h$ can be extended to a quasiconformal mapping of the lower half plane onto itself whose Beltrami coefficient $\mu$ is square integrable in the Poincaré metric, namely, $\mu \in M\left(\mathbf{U}^{*}\right)$. Without loss of generality, we may assume that $h(0)=0, h(1)=1$. By the well-known conformal sewing principle (see [Ah], [Le], [Na]), there exists a pair of quasiconformal mappings $f_{1}, f_{2}$ on the whole sphere $\hat{\mathbf{C}}$ which satisfies the following properties:

(1) Both $f_{1}$ and $f_{2}$ fixes the points 0,1 , and $\infty$;

(2) $f_{1}=f_{2} \circ h$ on the real line;

(3) $f_{1}$ is conformal in $\mathbf{U}$, with Beltrami coefficient $\mu_{1}$ in $\mathbf{U}^{*}$ being square integrable in the Poincaré metric, that is, $\mu_{1} \in M\left(\mathbf{U}^{*}\right)$;

(4) $f_{2}$ is conformal in $\mathbf{U}^{*}$, with Beltrami coefficient $\mu_{2}$ in $\mathbf{U}$ being square integrable in the Poincaré metric, that is, $\mu_{2} \in M(\mathbf{U})^{1}$.

By conjugating to the unit disk case, we conclude that both $f_{1}$ and $f_{2}$ are absolutely continuous on the real line. Consequently, $h=f_{2}^{-1} \circ f_{1}$ is absolutely continuous on the real line.

By Theorem 4.4 we conclude that $\left.\log f_{1}^{\prime}\right|_{\mathbf{U}} \in \mathcal{D}(\mathbf{U})$. On the other hand, it is well known that each element $\phi \in \mathcal{D}(\mathbf{U})$ has boundary values almost everywhere on the real line, and the boundary function $\left.\phi\right|_{\mathbf{R}}$ belongs to the Sobolev class $H^{\frac{1}{2}}(\mathbf{R})$ (see $[\mathrm{Zy}]$ ). We use $\log f_{1}^{\prime}$ to denote the boundary function of $\left.\log f_{1}^{\prime}\right|_{\mathbf{U}}$. Then $\log f_{1}^{\prime} \in$ $H^{\frac{1}{2}}(\mathbf{R})$. Similarly, $\left.\log f_{2}^{\prime}\right|_{\mathbf{U}^{*}}$ has boundary value function on the real line, denoted by $\log f_{2}^{\prime}$, also being in the Sobolev class $H^{\frac{1}{2}}(\mathbf{R})$.

Now from $f_{1}=f_{2} \circ h$ we obtain

$$
\log h^{\prime}=\log f_{1}^{\prime}-\log f_{2}^{\prime} \circ h
$$

which implies by Proposition 5.1 that $\log h^{\prime} \in H^{\frac{1}{2}}(\mathbf{R})$ as required.

Acknowledgements. The authors would like to thank the referee for a very careful reading of the manuscript and for several corrections.

\section{References}

[Ah] Ahlfors, L. V.: Lectures on quasiconformal mapping. - Van Nostrand, 1966.

[AG] Astala, K., and F. W. Gehring: Injectivity, the BMO norm and the universal Teichmüller space. - J. Anal. Math. 46, 1986, 16-57.

\footnotetext{
${ }^{1} M(\mathbf{U})$ can be defined in the same manner as $M\left(\mathbf{U}^{*}\right)$.
} 
[BA] Beurling, A., and L. V. Ahlfors: The boundary correspondence under quasiconformal mappings. - Acta Math. 96,1956, 125-142.

[Cu] CuI, G.: Integrably asymptotic affine homeomorphisms of the circle and Teichmüller spaces. - Sci. China Ser. A 43, 2000, 267-279.

[Fi] Figalli, A.: On flows of $H^{\frac{3}{2}}$-vector fields on the circle. - Math. Ann. 347, 2010, 43-57.

[GL] Gardiner, F. P., and N. Lakic: Quasiconformal Teichmüller theory. - Math. Surveys Monogr. 76, Amer. Math. Soc., Providence, RI, 2000.

[GS] Gardiner, F. P., and D. Sullivan: Symmetric structures on a closed curve. - Amer. J. Math. 114, 1992, 683-736.

[GR] Gay-Balmaz, F., and T. S. Rativ: The geometry of the universal Teichmüller space and the Euler-Weil-Petersson equation. - Adv. Math. 279, 2015, 717-778.

[Ha] Hamilton, D. E.: BMO and Teichmüller spaces. - Ann. Acad. Sci. Fenn. Math. 14, 1989, $213-224$.

[HWS] He, Y., H. WeI, and Y. Shen: Some notes on quasisymmetric flows of Zygmund vector fields. - J. Math. Anal. Appl. 455, 2017, 370-380.

[KP] Kufner, A., and L. E. Persson: Weighted inequalities of Hardy type. - World Scientific, Singapore, 2003.

[Le] Lehto, O.: Univalent functions and Teichmüller spaces. - Springer-Verlag, New York, 1986.

[Na] NAG, S.: The complex analytic theory of Teichmüller spaces. - Wiley-Interscience, 1988.

[NS] NaG, S., and D. Sullivan: Teichmüller theory and the universal period mapping via quantum calculus and the $H^{\frac{1}{2}}$ space on the circle. - Osaka J. Math. 32, 1995, 1-34.

[Po] Pommerenke, Ch.: Boundary behaviour of conformal maps. - Springer-Verlag, Berlin, 1992.

[Se1] Semmes, S.: Estimates for $(\bar{\partial}-\mu \partial)^{-1}$ and Calderón's theorem on the Cauchy integral. Trans. Amer. Math. Soc. 306, 1988, 191-232.

[Se2] Semmes, S.: Quasiconformal mappings and chord-arc curves. - Trans. Amer. Math. Soc. 306, 1988, 233-263.

[Sh] Shen, Y.: Weil-Peterssen Teichmüller space. - Amer. J. Math. 140, 2018 (to appear).

[ST] Shen, Y., and S. TANG: Weil-Peterssen Teichmüller space, II. - arXiv: 1801.10361.

[TT] Takhtajan, L., and L.-P. Teo: Weil-Petersson metric on the universal Teichmüller space. - Mem. Amer. Math. Soc. 183:861, 2006.

[Zh] Zhu, K.: Operator theory in function spaces. Second edition. - Math. Surveys Monogr. 138, Amer. Math. Soc., Providence, RI, 2007.

[Zhu] Zhuravlev, I. V.: A model of the universal Teichmüller space. - Sibirsk. Mat. Zh. 27, 1986, $75-82$.

[Zy] Zygmund, A.: Trigonometric series. - Cambridge Univ. Press, Cambridge, 1979. 